Research Article

\title{
Decisions on the Orderliness and Collaborative Operation Mechanism of Each Subsystem under the Organizational Model of the Internet of Things
}

\author{
Lingyi Cai $(\mathbb{D})$ and Wei Liu \\ School of Computer Science, Hubei University of Technology, Wuhan, Hubei 430068, China \\ Correspondence should be addressed to Wei Liu; 1910300628@hbut.edu.cn
}

Received 29 April 2021; Revised 25 May 2021; Accepted 1 June 2021; Published 10 June 2021

Academic Editor: Yi-Zhang Jiang

Copyright (c) 2021 Lingyi Cai and Wei Liu. This is an open access article distributed under the Creative Commons Attribution License, which permits unrestricted use, distribution, and reproduction in any medium, provided the original work is properly cited.

\begin{abstract}
This paper analyzes the overall organic network system integrated by subnetworks, analyzes the network evolution process of the overall system of the Internet of Things (IoT) organization mode network, and analyzes the network through the network scale measurement, the node growth measurement, the node distribution measurement, and the node association measurement. We analyze the coordination mode of hardware resources, information resources, financial resources, human resources, organizations, and customer resources from the perspective of resource elements. And we analyze the vertical and horizontal work collaboration mode from the perspective of work activities. From the perspective of equity, two types of equity-based synergy and four types of contract-based synergy are proposed. It is proposed that the network stage of the IoT organization model is different from the previous stage of the important collaboration model, the platform-based collaborative model, which is analyzed from the coordination manager software platform and the IoT organization model infrastructure hardware platform. We use relevant data to construct a SIM model to measure the synergy of the development of the IoT industry system to quantify the overall collaborative development status of the IoT industry system. At the same time, the gray correlation analysis model GM $(1, N)$ is used to correlate the complex system subsystems. The research results show that the development of the IoT industry has gone through a U-shaped development process from the uncoordinated stage to the coordinated development of the industrial system. Each subsystem has self-development capabilities and exhibits different interaction relationships with each other.
\end{abstract}

\section{Introduction}

The Internet of Things (IoT) is based on communication networks such as the Internet and mobile communication networks [1]. According to the application needs of different industries, it uses the perception, communication, and computing capabilities of agents to automatically obtain real-world information and connect all physical objects with independent addressing capabilities, so as to build an intelligent information service system that connects people and things and things and things. The IoT in specific application areas (smart agriculture, smart cities, etc.) still mainly stays in the links of environmental information perception, data transmission, and monitoring [2]. Although sensor technology is used, most of the collected data are used for display or statistical analysis, and there is no information linkage with related control equipment. In fact, scientific decision-making and intelligent control are not realized. The more common applications are perception without decision-making or decision-making without control. These applications are single-step applications, which have not yet constituted the "closed loop" of the entire link of "sensing-decision-control," which greatly weakens the IoT technology [3].

Under the new economic form, there are new development forms of the IoT organization model and development needs of the IoT organization model. Scholars supplement and improve the theoretical system of the organization model of the IoT, combine the current economic development status and trends, make new interpretations 
and more in-depth studies, and propose the network system planning and operation methods of the organization model of the IoT on this basis $[4,5]$. It is of great theoretical significance to enrich and develop the theoretical system of the subject of the organizational model of the IoT. The network construction optimization model studied in this paper, as well as the network coordination operation mode and coordination mechanism, realizes the dynamic sharing of information and resources of the main body of each dimension in the network, the coordination and integration of operation processes, and better specialization and integration of services for regional economic industries. It is expected to provide scientific methods and basis for the Chinese government and enterprises in the IoT organization model planning, promote the development of the IoT organization model industry cluster, optimize the overall operating performance of the network system, reduce the overall operating cost of the IoT organization model network system, optimize IoT organization model network resource allocation, reduce process links and time-consuming, improve the degree of cooperation and coordination of the IoT organization model network system, promote the increase of the value of the IoT organization model, and have practical guidance for promoting the development of the IoT organization model industry significance [6].

Coordinating managers can exist stably and effectively promote collaborative management work and must pass a series of institutional guarantees and government support. First, the establishment of a coordinated management organization needs to be led by relevant government departments, with the participation of key IoT organizations and park management committees. Then, it is responsible for formulating and implementing the system's task coordination execution mechanism, information sharing mechanism, collaborative incentive mechanism, and collaborative behavior. The supervision data are obtained by means of information, and the reward and punishment results and early warning information are output through the established mechanism model to assist in the implementation of the mechanism. Based on the measurement of the degree and status of the development of the IoT industrial system, a gray dynamic correlation analysis model of the synergy among the various subsystems within the IoT industrial system, namely, the GM $(1, N)$ model, is constructed according to the gray system theory. At present, each subsystem has the ability to develop itself. Among them, the fusion subsystem and the technological innovation subsystem have a one-way weak synergy relationship.

1.1. Related Work. The IoT is a very complex heterogeneous network, which builds a bridge between the physical world and the virtual world [7]. The ultimate goal of all IoT applications is to provide seamless services without human intervention. The IoT is considered to be the next logical revolution, which can provide a wide range of services in smart cities, manufacturing, smart agriculture, smart healthcare, and smart homes [8, 9]. Autonomous IoT systems are very important, but there are still many challenges to be solved. Stankovic mentioned in the inaugural IEEE "Journal of the IoT" that the IoT should be an important infrastructure that can run multiple applications and services and has integration capabilities [10]. Scholars proposed a kind of cognitive IoT, including how to deal with heterogeneous data and high-dimensional data of the IoT, the discovery of the semantics of the IoT, and the interpretation of related technical issues such as the intelligent decisionmaking of the IoT and in-depth research on the intelligent services of the IoT. The IoT has quietly entered multiple smart industries. In today's advocacy of energy conservation and environmental protection, the optimization of the resource allocation of the IoT has become an urgent problem to be solved [11]. There are many artificial intelligence algorithms for resource scheduling, but most of them are developed for specific applications and have certain limitations, which are not suitable for solving the IoT service problems studied in this paper [12]. As the IoT is a service, this paper will treat its entire layout as a service system. The solution of the resource optimization allocation problem of service-oriented networked collaborative equipment is a very complex problem, which belongs to the typical NP-hard combinatorial optimization problem [13]. The question studied in this paper is how to reduce resource consumption and shorten service time, in other words, how to select multiple optimal services from a large set of candidate services to meet the above goals. This will be a challenging multiobjective optimization problem.

Many researchers try to solve the multiobjective service selection problem in Web services [14]. Scholars proposed the first approximation of multiobjective quality-driven service selection [15]. Related scholars have carried out research on multiobjective optimization of service quality. They introduced the Pareto set model for service composition with service quality perception. Related scholars have proposed a method to support decision-makers to use clustering to find robust and QoS-optimized service combinations [16]. In our previous research work, we implemented an adaptive Web service composition inspired by the neuroendocrine-immune system. However, the abovementioned research work mainly focuses on Web service composition based on service quality. IoT services is different from Web services in that they are characterized by large-scale, heterogeneous, unreliable, and dynamic nature. An important challenge that needs to be solved in the field of IoT service composition (also known as service integration) is to develop efficient service selection algorithms in order to optimize management of energy consumption and service quality. In a large-scale IoT environment consisting of thousands of distributed entities, this issue becomes critical. Relevant scholars use probabilistic discovery methods to efficiently find an approximate service set that satisfies a given request to meet the goals of rapid service discovery and minimize resource consumption [17]. Related scholars pointed out that the IoT is a paradigm, in which physical things in the real world can be connected to the Internet and provide services through additional computing devices. Researchers have proposed a three-tier service quality scheduling model for the service-oriented IoT. Perception as 
a service model is expected to be built on IoT infrastructure and services. Then, the service is allocated to the interface with heterogeneous resources, and the optimal solution is generated for this NP-hard problem [18].

The large-scale IoT environment is composed of thousands of distributed entities. Once a service request is detected, it is necessary to immediately calculate and select multiple (request-service) services with the optimal combination of request-service pair from a large number of candidate sets to provide the service. The IoT should not only provide services for dynamic concurrent requests but also reduce energy consumption, reduce service time, and improve information accuracy. In addition, service availability, bandwidth allocation, and reliability should also be considered, especially in resource-constrained environments [19]. Therefore, service selection is a multiobjective optimization problem. However, the development of effective service selection algorithms is still challenging and has not been extensively studied. Recent research results, such as the use of novel decomposition strategies, heuristic caching strategies, and heuristic multitask scheduling algorithms, are applied to the IoT service selection problem, but they have not been in-depth from the perspective of multiobjective optimization [20]. The optimization of IoT service portfolio based on resource and QoS awareness and the optimization of IoT service portfolio based on energy consumption and QoS achieve dual-objective optimization, but the dynamic characteristics of the service are not considered [21, 22]. Therefore, the purpose of this paper is to achieve global combination and multiobjective optimization of ubiquitous services in a dynamic IoT environment.

\section{Core Technology for Decision- Making Collaboration}

2.1. The Process of Decision-Making Coordination. The ultimate goal of autonomous decision-making is to better conduct penetration testing, that is, detection, intrusion, and information theft of the target system. After obtaining important information, it is necessary to use the same channel and encryption method as legal data for information transmission through controlled distributed clients and disguised applications, concealing itself while avoiding security audits and anomaly detection mechanisms.

Effective and reasonable attack decision-making will realize the migration of multiple objects. Therefore, autonomous decision-making needs to have a clear recipient object and target (including attack range, target effective value, attack time limit, and damage degree) at specific stages, the system type, defense mechanism, and security equipment deployed are comprehensively planned, and the data collected in the information collection phase are dynamically adjusted during the simulation attack phase to achieve the optimal attack effect, transfer to the next object, and finally achieve the target system penetration effect.

This paper mainly involves the decision-making system and collaboration system, as well as the later development of the penetration test module. The whole working process is shown in Figure 1.
The whole system includes three stages: decision-making stage, collaboration stage, and penetration testing stage. The decision-making stage includes four main functional modules, namely, authentication module, node selection module, task expression, and task segmentation. The collaboration stage is divided into functional modules such as task distribution, idling, communication, and exit according to the inherent needs of the collaborative system and the process of collaborative processing.

2.2. Task Segmentation. In order to increase the effectiveness of decision-making, the task constraint conditions set up are expressed with the constrained object as the center. For example, in the decision-making authentication stage, there are restrictions on authentication information, including key/certificate information, version information, and so on. The target object usually includes constraints such as operating system, network environment, and application environment. It is necessary to divide the task into basic executable tasks. When the task is a compound task, the "task segmentation and scheduling" must be completed before the task is executed.

In order to achieve the balance of the system and simplify the algorithm, it mainly examines the hardware conditions of the node such as CPU, disk, and memory, as well as the network information and operating system version of the node. These characteristics are combined to make node selection. Different nodes are selected for the decision tasks of different target hosts. For example, when sniffing and scanning the hosts in the same local area network, it requires less resources, but higher requirements are placed on the network of the node. At this time, the network information and other parameters of the node are mainly used as the basis for selection. When the task becomes a password cracking that requires more hardware resources such as $\mathrm{CPU}$ and memory, the node's choice of focus is converted to the node's hardware as the main reference basis. This is because password cracking attacks often require a lot of local resources. Of course, network resources have also been improved. Therefore, when designing the node selection algorithm, we need to combine tasks for node selection. For this, you can refer to the task type after task segmentation in the previous section to set the corresponding selection coefficient.

2.3. Cooperative Communication. The design of the cooperative communication module is for the communication between cooperative nodes and between cooperative nodes and cooperative controllers. The protocol interaction method involves the communication between the two ends of the C/S. Channel is used to maintain the "channel" information, including the SOCKET (abstract description) of the communication and the option information of the SOCKET. The design is based on the support of multiple operating system platforms. By maintaining the attribute information corresponding to the socket handle, using Channel can facilitate other modules to modify these attributes and increase these interfaces. After combining the 

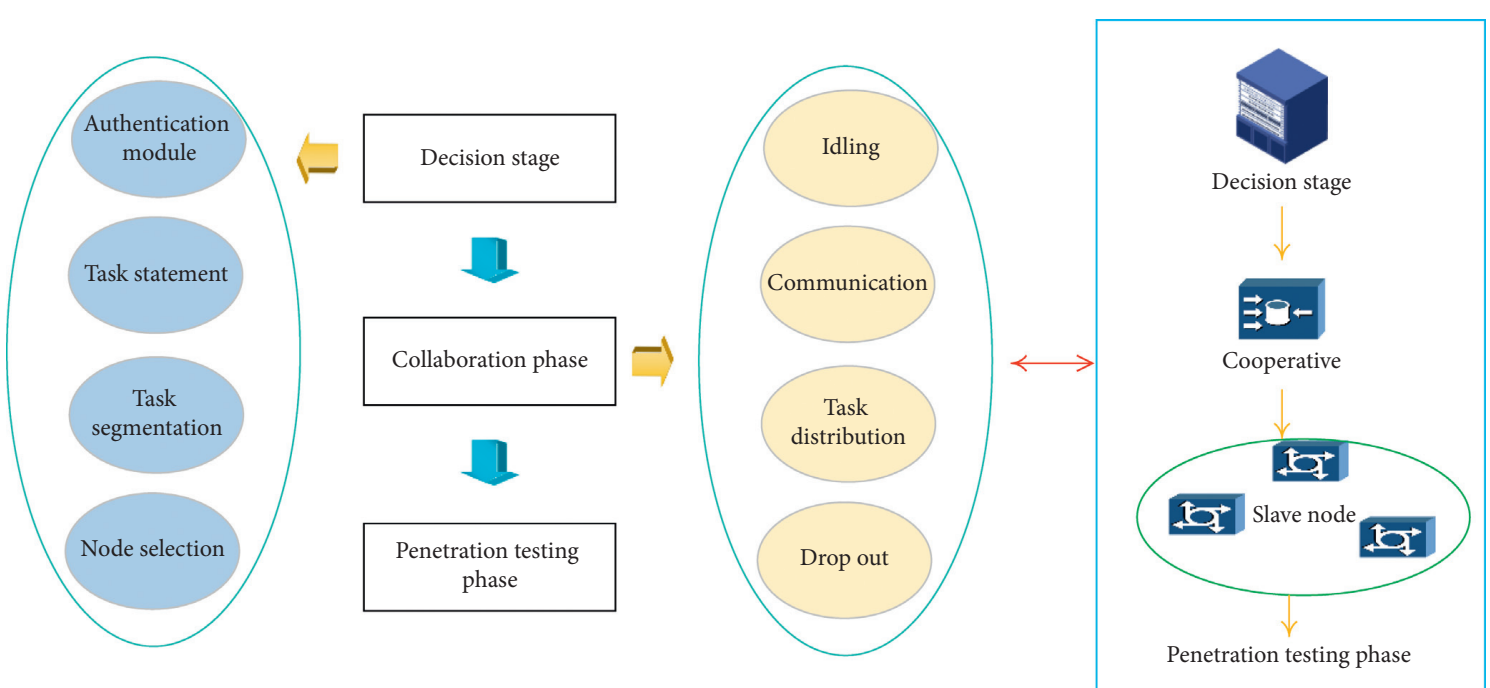

FIGURE 1: System working process.

data management, the corresponding implementation results should meet the model diagram shown in Figure 2.

\section{Decisions on the Collaborative Operation Mechanism of the Network System of the Multidimensional IoT Organization Model}

3.1. The Principle and Process of the Orderly Cooperative Operation Mechanism of Each Subsystem. The collaborative operation of the IoT organization mode network is a series of IoT organization mode activities that are oriented to customer needs and revolve around the customer's IoT organization mode service tasks. In the IoT organization model network system, through the coordination management platform, $m$ customer tasks are accepted and decomposed into $n$ IoT organization model operations, and the paths of these operations are optimized and integrated. You collect $k$ IoT organization model service providers that can provide service resources through the network IoT organization model platform and evaluate and match operation resources. From the overall perspective of the system, you call the resources in the network system, select the optimal configuration of the resources, and work together to complete the tasks of the IoT organization mode by the division of labor selected by these preferred resources. This collaborative operation mechanism is based on customer needs. The mechanism enables the resource distribution of the IoT organization model service provider to be guided by the overall operation requirements of the system, continuously optimizing the distribution and migration and realizing the optimization of the overall system operation.

The collaborative operation mechanism of the IoT organization model network is the core of all other collaborative mechanisms. Node members can carry out collaborative behaviors such as division of labor and cooperation in accordance with the rules of the collaborative operation mechanism and then can further implement the incentive and supervision mechanism of collaborative behavior on this basis. The collaborative operation mechanism studies how to integrate operations and achieve economies of scale and distance economic effects and how to match resources to give full play to the respective advantages of the IoT organization model service providers, reduce vicious competition, and reduce the return load rate, thereby reducing the overall cost of the network. This process mainly includes the three processes of job grouping, job integration, and resource matching execution. The rules involved in the three stages include main job sorting rules, job integration rules, and resource matching selection rules, as shown in Figure 3.

In the node operation, the operations of the same location and the same type are integrated. The integration of transportation operations is more complicated. If the scale effect is not considered, each operation chooses to take the shortest path between the starting point and the end point, but it may lose the opportunity to integrate with other operations. It is not necessarily the overall best choice. In the same group of transportation operations, according to the evaluation of the main operations from high to low, the shortest paths of the operations are found in turn, and the nonmain operations are integrated into the path of the main operations in order to achieve economies of scale. The step is to list the shortest path of each job. The Dijkstra algorithm can be used, and the number of iterations is set to K; that is, $\mathrm{K}$ shortest path solutions are listed. The second step is to integrate the operation path and determine which operations can be integrated into the main operation path. If the cost saved by the scale effect can offset the detour cost and transshipment cost, then integrate; if the increased cost cannot be offset, then do not integrate.

We match the integrated work according to the resources of the nodes and lines and find the resources in the path according to the previous path plan. According to the resource situation of the service provider, it can be completed by a single service provider or multiple service providers. This process is effective recombination of the 

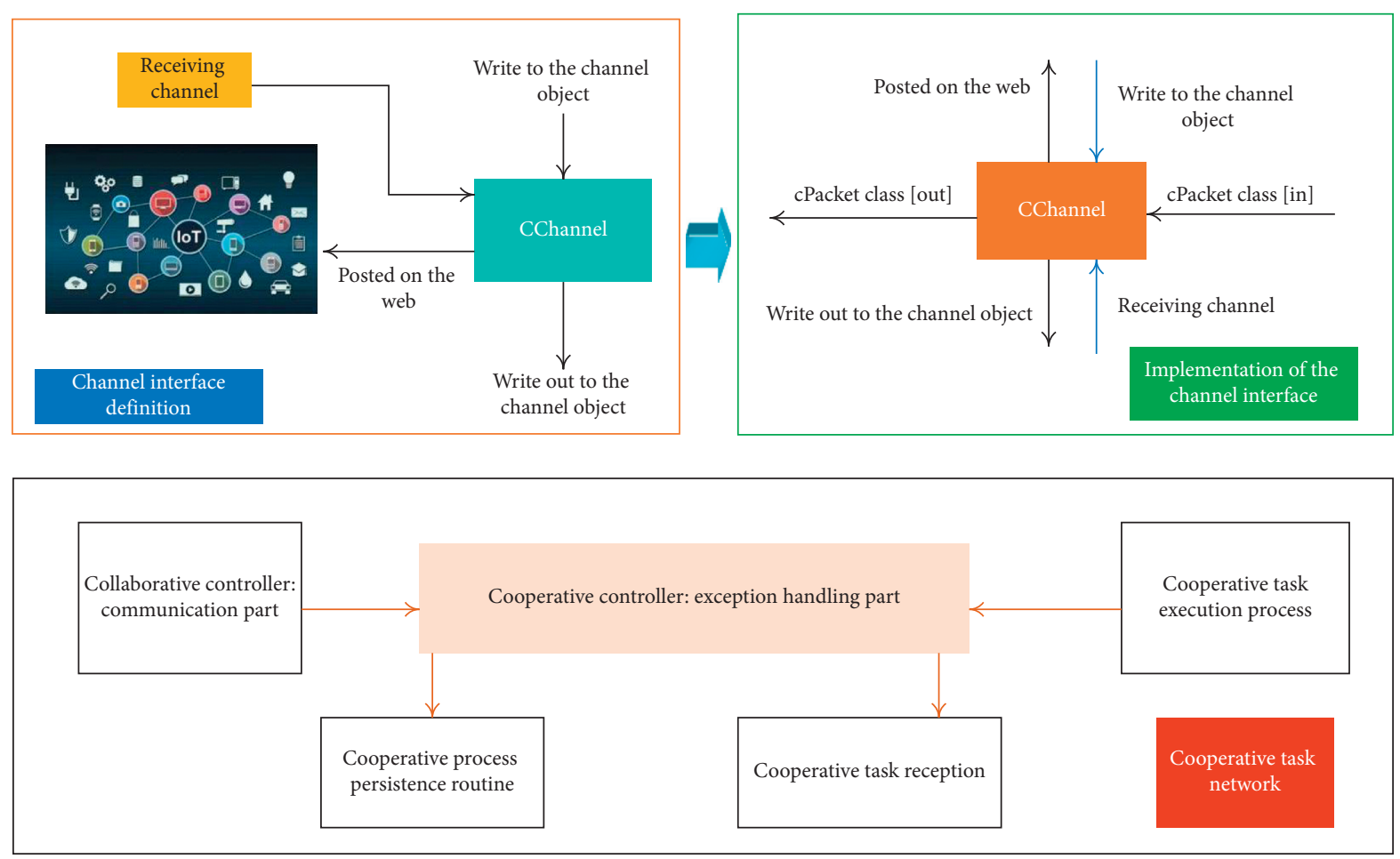

FIGURE 2: Schematic diagram of the model after the channel interface is implemented.

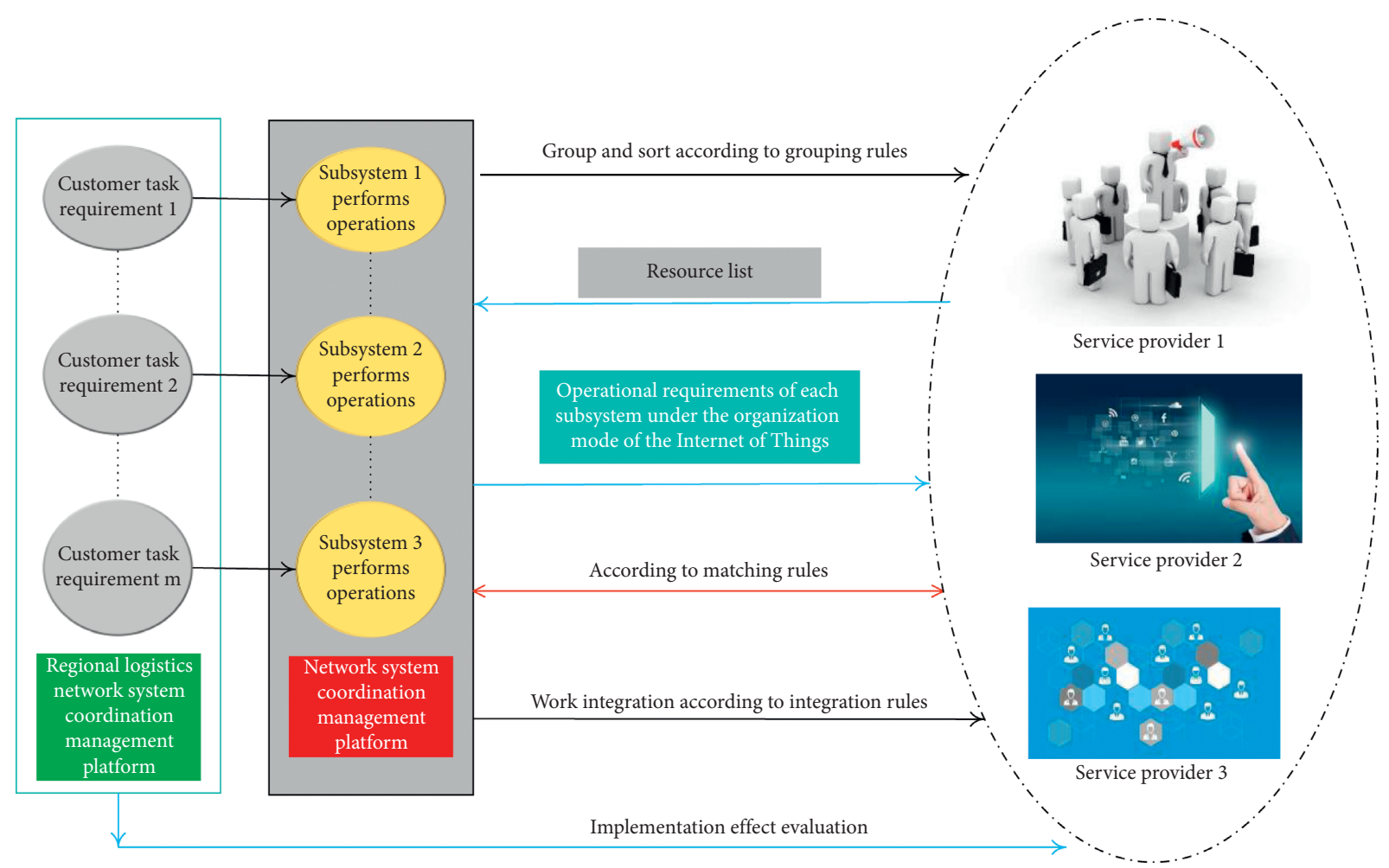

FIgURE 3: The collaborative work process of the IoT organizational model network.

service resources of the distributed IoT organization model. It is the redesign of the organization model network of the IoT organization model to quickly respond to market demand for its own process and organization.
According to the published service requirements, the resource search is performed according to the search conditions, and the information of the candidate companies that match the conditions is displayed in feedback. You give 
priority to the resources and success history of the service provider and then judge whether the resources can complete the task. When the working capacity $\mathrm{Ai}$ of the preferred service provider is sufficient, the job Ti of the node or the line can be completed independently. When the existing resources of the service provider cannot meet the demand, the suboptimal service provider is selected in turn to subcontract to complete the operation. If the total resources of the service provider at this point (route) are still insufficient, you apply to call the idle resources of the service provider of other nodes (routes).

\subsection{Establishment of an Orderly Collaborative Operation} Mechanism Model for Each Subsystem. Suppose that the network graph of the IoT organization model is composed of points and edges, $G=(V, L)$, where $V$ is the set of nodes, $m$ is the total of points, and $L$ is the set of edges. $G$ becomes the abstraction of the nodes and traffic connections of the IoT organization model in the geographic map. Due to the limitation of transportation capacity of service providers, each service provider can only transport part of the road sections in the transportation network. Therefore, the network diagram of the IoT organization mode is a mixed road network diagram, and the side variable $Y\left(v_{i}, v_{j}\right)=S_{k}(k=1$, $2, \ldots, K)$, where $S_{k}$ represents the service provider, indicating that the edge $\left(v_{i}, v_{j}\right)$ is the transportation route of the service provider $S_{k}(k=1,2, \ldots, K)$, and the point variable $B$ $\left(v_{i}\right)=S_{k}(k=1,2, \ldots, K)$, indicating that vertex $v_{i}$ is the job node of the service provider $S_{k}$. $R_{i j}$ is the number of service providers between points $i$ and $j . S_{i j k}$ is the $k$-th service provider between points $i$ and $j$.

Suppose that there are $N$ jobs in the network to form job set $Z ; \mathrm{Zn}$ ( $n, \mathrm{Va}, \mathrm{Vb}, \mathrm{qn}, T_{\max }, C_{\max }$, type) means that the starting point of the nth job $\mathrm{Zn}$ is $\mathrm{Va}$, the end point is $\mathrm{Vb}$, and the amount of work is qn. For time requirements, $C_{\max }$ is the cost budget of the customer; that is, the time for customer satisfaction cannot exceed $T_{\max }$, and the cost cannot exceed $C_{\max }$. Type represents the job type and is used for the group number of the job.

Suppose that there are a total of $K$ service providers in the network to form a service provider set $S$. $S_{k}$ means that the operation capacity of the $k$-th service provider $S_{k}$ between the service range $V_{i}$ to $V_{j}$ is $A_{i j}$, the total amount of work undertaken is $Q_{i j}$, ijn the ability to undertake the service is evaluated as $D_{i j}$, the service price $C_{n}$ represents the price required to complete the transportation operation of $\mathrm{Zn}, C_{n}^{\prime}$ represents the price required to complete the node operation $\mathrm{Zn}, T_{n}$ represents the completion of the transportation operation, $T_{n}^{\prime}$ represents the time required to complete the node job $\mathrm{Zn}$, and type is the resource type of the service provider, which matches the type grouping in the job variable.

$$
\begin{aligned}
Q_{i j k} & =\prod_{n=0}^{N-1} y_{n i j k(R n)} \bullet q_{n-1}, \\
C_{n} & =q_{n-1} \bullet L_{i j} \bullet C_{i j} \bullet Q_{i j} \\
C_{n}^{\prime} & =q_{n-1} \bullet C_{i j}^{\prime} \bullet Q_{i j} .
\end{aligned}
$$

Among them, $C_{i j}\left(Q_{i j}\right)$ is the price per unit of distance per unit of work, which is a descending function or a segmented discount function. The larger the total amount of work undertaken by the service provider $S_{k}$ in this segment, the lower the price per unit of work, which reflects the effect of scale.

$$
C_{i j}\left(Q_{i j}\right)= \begin{cases}\alpha_{1} c_{i j}, & Q_{i j}>b, \\ \alpha_{2}\left(1-c_{i j}\right), & a<Q_{i j}<b, T_{n}=\frac{\left(L_{i j}-1\right)}{\left|V_{i j}\right|}, T_{n}^{\prime}=\frac{\left(q_{n}-1\right)}{\left|V_{i j}^{\prime}\right|} . \\ c_{i j}, & 1<Q_{i j}<a,\end{cases}
$$

3.3. The Process of Solving the Orderly Coordination Mechanism of Each Subsystem. The two-layer iterative method is used to solve the problem, and the solving process is shown in Figure 4. The solution is divided into two levels. The first level is job reorganization. First, the main job-based job boarding method is used for integration and optimization, so that resource matching is guided by system optimization, and the service provider selection is the second level problem. The suboptimization problem of the problem can be solved by using the neighborhood search method.

3.4. Establishment of an Incentive Mechanism Model for Orderly Coordinated Behavior of Each Subsystem. The idea of this mechanism model is mainly to further improve on the basis of the price management mechanism model and carry out collaborative gain distribution. The synergy gain is the difference between the system's optimal return Etotal * and the noncooperative total return Etotal $* *$. It is assumed that a part of the gain will be distributed to all members of the IoT organization model service provider participating in the collaboration, and the other part will be rewarded to the IoT organization model service provider with excellent service and active network construction behavior. That is to say, the income of the service provider is divided into the basic subcontracting income plus the reward income.

Suppose that there are K IoT organization model service providers in the IoT organization model network system, the annual subcontracting operation volume of the kth IoT 


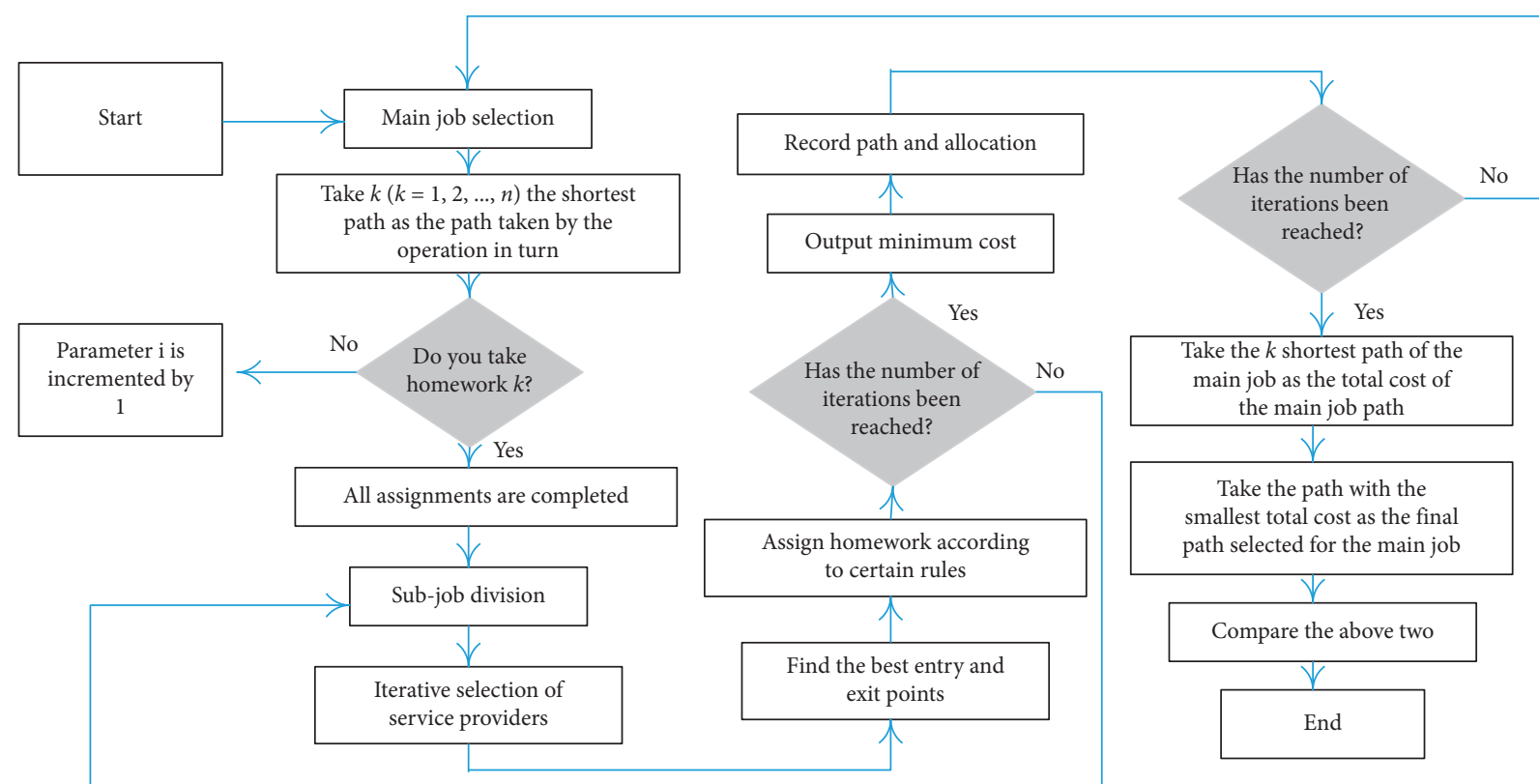

FIGURE 4: Overall solution process of orderly coordination mechanism of various subsystems.

organization model service provider is $\mathrm{qk}$, and the operation execution result is $\mathrm{Uk}=(\mathrm{uk} 1, \mathrm{uk} 2, \mathrm{uk} 3) \mathrm{T}$. Among them, uk1 is the basic IoT organization mode operation assessment, including on-time arrival rate and customer service evaluation. uk2 is the information construction index of the organization model of the IoT, such as the timely rate of information online, the coding rate of equipment standardized information, and the investment in information construction such as EDI. uk3 is the coordination and cooperation index with other IoT organization model service providers, such as the completion rate of loading and unloading preparation and the timely rate of vehicle preparation; before the arrival of the downstream subcontractor's transport vehicle, we prepare for shipment. These three types of evaluation indicators are integrated into the 360-degree inspection method; that is, the company itself, customers, the coordinator of the IoT organization model platform, and the upstream and downstream cooperative companies jointly evaluate. Most of the evaluation information is automatically entered into the calculation method based on objective work conditions to reduce management costs brought by incentives. The subcontract of the service provider to improve the organization model of the IoT is

$$
S_{k}\left(U_{k}\right)=\prod_{i=0}^{2} q_{k} \bullet \alpha_{k i}\left|u_{k i}-u_{k i 0}\right| \bullet p_{k-1}, \quad k=1,2, \ldots, n .
$$

Among them, the first item is incentive payment, and the second item is fixed standard payment. $u_{k i 0}$ is the up-tostandard value of each index, and $\alpha_{k i}$ is the incentive coefficient of each index, which is also the decision variable to be solved in this section. $p_{k}$ is the fixed payment for completing the job task and meets the $P_{i}$ interval range of the previous section; set

$$
\left\{\begin{array}{l}
u_{k 1}=e_{k 1}-\left|\varepsilon_{k 1}\right|, \\
u_{k 2}=e_{k 2}-\left|\varepsilon_{k 2}\right|, \\
u_{k 3}=e_{k 3}-\left|\varepsilon_{k 3}\right| .
\end{array}\right.
$$

Among them, $\varepsilon_{k i}(i=1,2,3)$ represents the effort spent by the k-th IoT organization model service provider in the i-th type indicator. $\varepsilon_{k i}(i=1,2,3)$ represents the external objective factors that affect the completion of the i-th type index of the k-th IoT organization model service provider. The external objective factors that affect the operation index of the IoT organization model include weather conditions, traffic conditions, and natural disasters; external objective factors that affect information sharing include the service conditions of public telecommunication operators and the information network construction of the IoT organization model network platform.

Suppose that the benefits brought by the $j$-th IoT organization model service provider to the IoT organization model network system are

$$
H_{k}\left(U_{k}\right)=\prod_{i=0}^{2} q_{k-1} \bullet u_{k i} \bullet \beta_{k i} .
$$

Among them, $\beta_{k i}$ reflects the contribution of the i-th index to the system revenue. The first category of indicators is the completion of the organization model of the IoT, the second category of indicators is the status of information construction and sharing, and the third category of indicators is the status of coordination and cooperation.

Suppose that the coordination cost between the network platform coordinator of the IoT organization model and the $k$-th IoT organization model service provider is

$$
C_{k}=\frac{1}{k} \cdot \prod_{i=0}^{2} e_{k i}^{2}
$$


Therefore, the total benefits of all IoT organization model service providers in the IoT organization model network system to the coordinator are

$$
G=\prod_{k=0}^{K-1} q_{k-1} \bullet\left|S_{k}+C_{k}-H_{k}\right|
$$

\section{Evaluation and Analysis of the Synergy Degree of the IoT Industry System}

4.1. Determination of Indicator Weights. The degree of influence of each subsystem in the evaluation index system on the overall system is not the same, which requires the corresponding weight to be assigned to each evaluation index. This paper uses the correlation matrix weighting method to determine the indicator weights. The correlation coefficient reflects the degree of interaction between indicators. The larger its absolute value, the closer the relationship between the indicators. The deeper the impact, the lower the converse. If the overall correlation between an indicator and other indicators in the evaluation system is relatively high, it means that this indicator has a greater impact on the development and changes of other indicators; that is, it has a greater role in the system. Assuming that the index system contains $n$ indicators, the correlation matrix between the indicators is represented by $R$; let

$$
R_{j}=\prod_{i=0}^{n-1}\left(1-r_{i j}\right), \quad i=0,1, \ldots n-1 .
$$

Then $R_{i}$ represents the total impact of the $\mathrm{i}$-th indicator on other $n-1$ indicators in the indicator system. The larger $R_{i}$, the greater the impact of the i-th indicator in the indicator system and the greater its weight. The corresponding index weight can be obtained by normalizing $R_{i}$; namely,

$$
\lambda_{i}=\frac{R_{i}}{\prod_{i=0}^{n-1} R_{i}}, \quad i=0,1, \ldots, n-1 .
$$

The calculated weight of each evaluation index and the proportion of each index are shown in Figure 5.

4.2. Measurement and Evaluation of the Synergy Degree of the IoT Industry System. The coordination degree of each subsystem of the IoT industrial system and the coordination degree of the IoT industrial system are calculated. The weights of the four subsystems are all set to 0.25 when calculating the coordination degree of the composite system. The estimated results are shown in Figure 6.

Figure 7 shows the degree of synergy among the various subsystems of the IoT industry system. It can be seen from the figure that the coordination status of each subsystem is more complicated. The degree of synergy of the fusion subsystem is rising in volatility, and after reaching the peak, it shows a downward trend to the lowest point. The decline in the degree of synergy of the fusion mechanism shows that the industrial barriers have not been eliminated, and it is still hindering the coordinated development of the IoT industry.
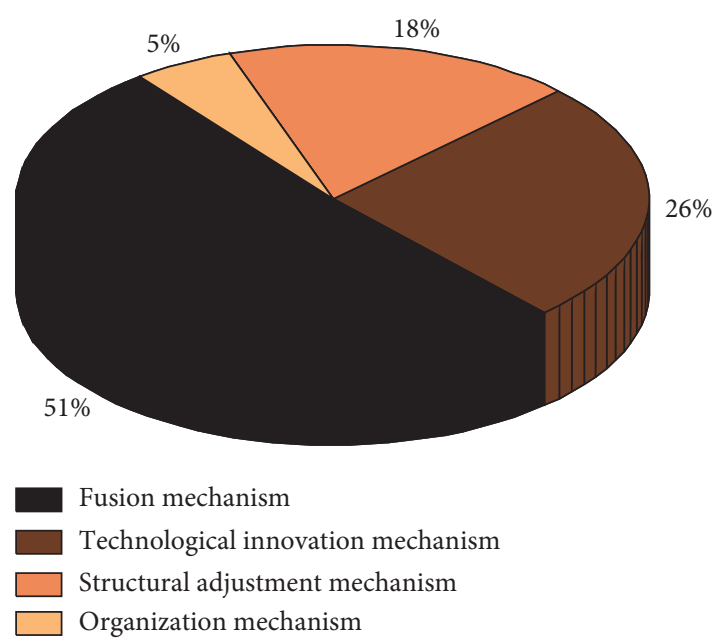

FIgURE 5: Proportion of the evaluation index of the synergy degree of the IoT industry system.

After that, it showed a trend of picking up again, indicating that industrial integration has gradually developed slowly after experiencing a process of disorderly development. The reason is that the IoT industry chain is long, and the differences between different industries have hindered product interaction to a certain extent. Effective communication between the information collected by different industries in this layer is different, so the available sensors are different. For example, the sensors used for soil monitoring and the sensors used for environmental monitoring are not universal. The same is true at the information processing level. The content they need to analyze and make decisions about is different from each other. In the current period of development, due to the diversified nature and strong customization of the industry chain including industries, it is difficult for IoT technology and industry growth to form a centralized leapfrog development. After reaching the peak point, the organizational mechanism synergy showed a downward trend, indicating that the organizational mechanism that can lead the development of the IoT industry has not yet been perfected.

The growth synergy degree of the IoT industry system is shown in Figure 8. From the perspective of the degree of synergy of the growth system of the IoT industry, the development and growth of the IoT industry have gone through a wave-shaped development process from the uncoordinated development of the current industrial system. IoT-related companies are generally small in scale and weaker in resisting risks and are more affected by the financial crisis. On the other hand, since the development of the IoT was proposed, it has brought opportunities and problems to the development of the IoT. With regard to the IoT, scientific research institutions and local governments have begun to rush to get a share of government investment. This has caused a serious situation of low-level redundant construction. The future development of the IoT has a broad space, but it is separated by many different application scenarios, forming a large number of small markets with limited capacity, which has caused a phenomenon that 


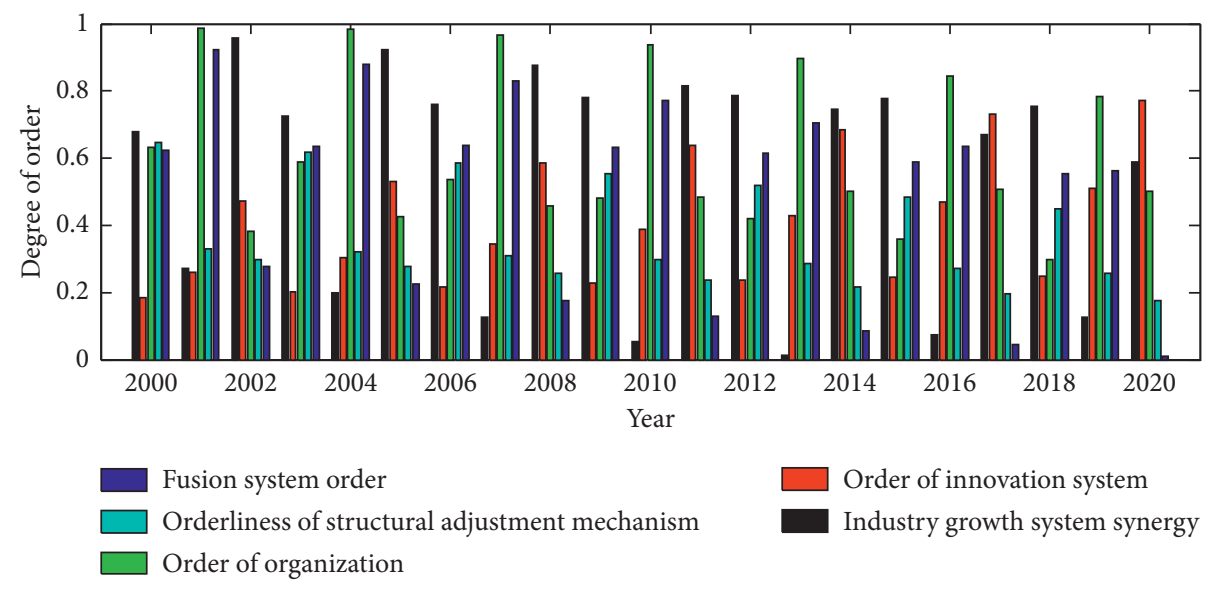

FIgURE 6: Estimation of the degree of synergy in the IoT industry system.

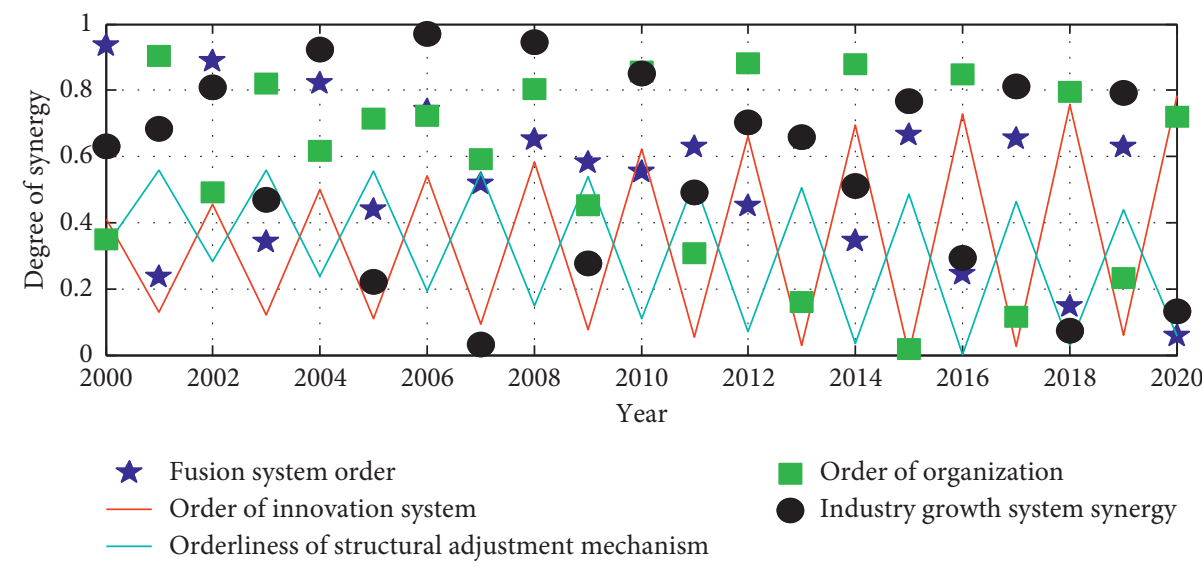

FIGURE 7: Changes in the degree of synergy among the various subsystems of the IoT industry.

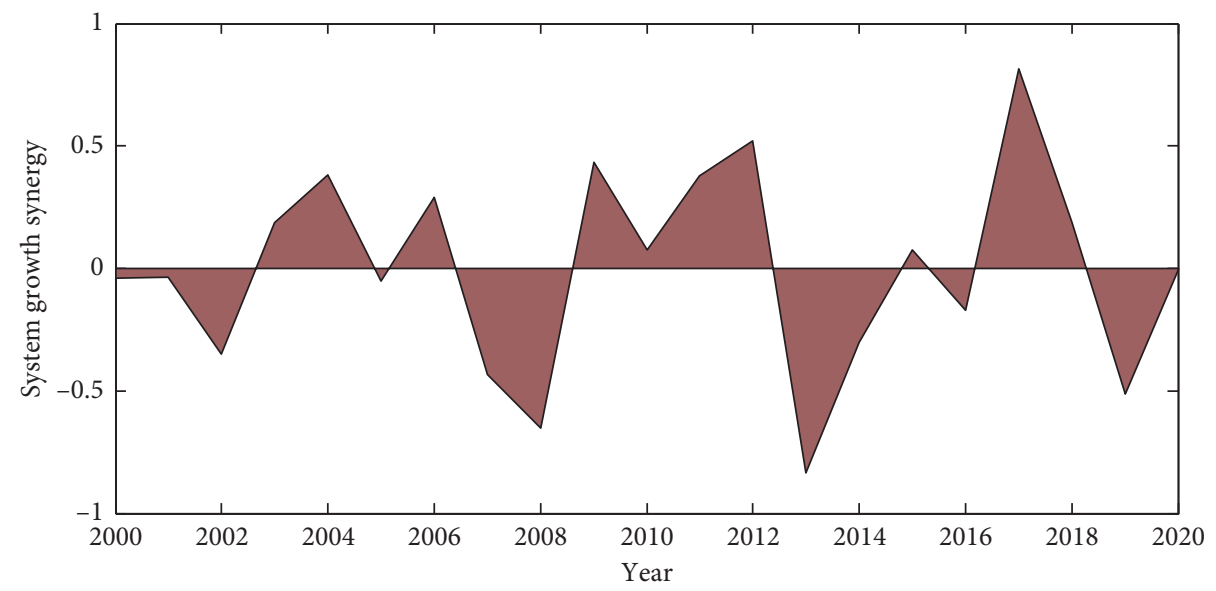

FIGURE 8: The degree of synergy in the growth of the IoT industry system.

diversified application scenarios limit the development of industrialization. This phenomenon is mainly manifested in two aspects. On the one hand, in the initial stage of the development of the IoT, the technology application and product development are not yet mature, and some of the IoT technologies are relatively high-end, and they lack advantages in price, which cannot satisfy the market's demand for product functions. It is difficult to obtain product promotion and large-scale development due to the requirements of sex and price; on the other hand, some of the current technologies cannot be used to create value and function by realizing productization. Now they only stay at 


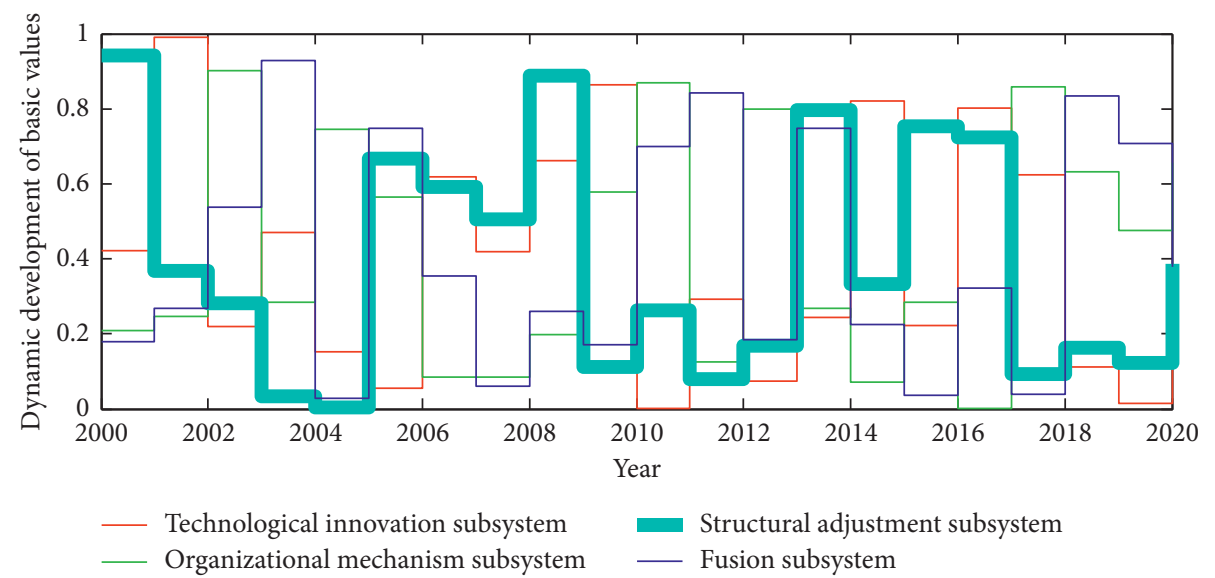

Figure 9: The basic data sequence of the dynamic development of each subsystem of the IoT industry.

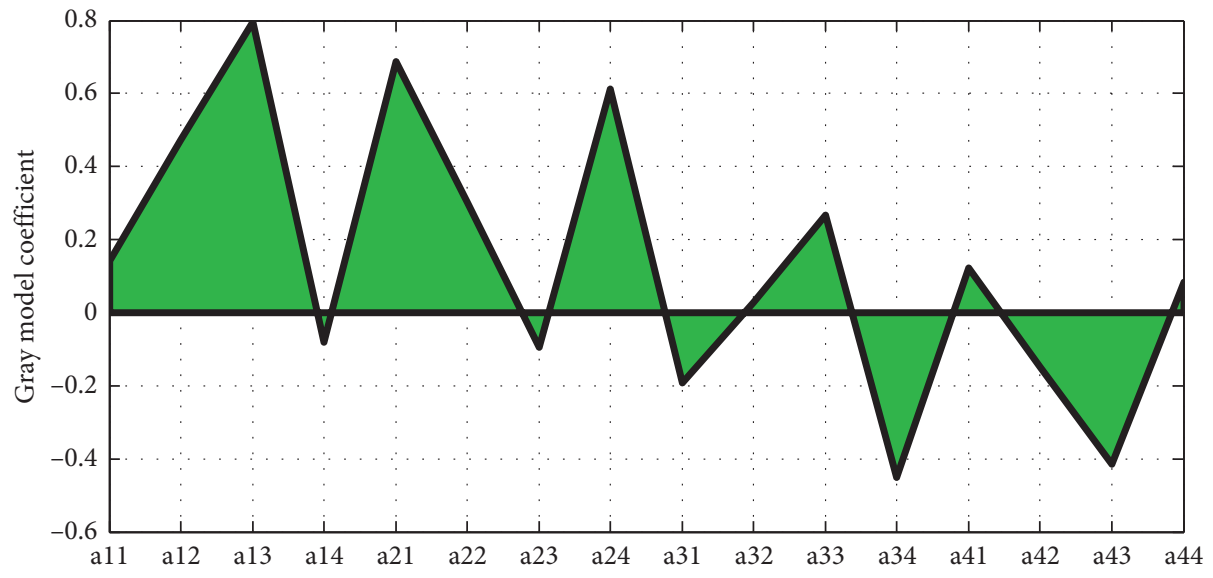

Figure 10: Gray model coefficients of various subsystems of the IoT industry.

the level of laboratory and simulation, which is difficult to form. Large-scale applications cannot promote the improvement of the industrialization level. For example, based on the application of sensors and sensor networks, the conflict between application scale and industrialization is very obvious. For example, the country does not have the ability to produce sensors for detecting soil and water quality. From the comparison of the order degree of the four subsystems, it can be seen that the organizational mechanism changes more frequently and the order degree shows a downward trend, which is the main reason why the IoT industry cannot develop in a coordinated manner. The development of industrial system synergy has contributed a lot.

\subsection{Evaluation of the Collaborative Development Relationship} of the Various Subsystems of the IoT Industry. On the basis of the overall collaborative measurement analysis of the IoT industrial system, a dynamic correlation analysis model of the synergy of the various subsystems within the IoT industrial system is constructed according to the gray system theory, namely, the GM $(1, \mathrm{~N})$ model.
4.3.1. Establishment of the Basic Data Sequence of Gray Evaluation of the Industrial System of the IoT. Based on the index data of the four subsystems of the IoT industrial system, this paper establishes the basic index data column of the gray system dynamic evaluation model. Based on the calculation of the index weights above, the dimensionless development sequence values of the four subsystems are calculated. This is the basic data column of the model. According to the basic data sequence method of establishing the gray dynamic evaluation model, the basic data sequence of the development of each subsystem of the IoT industry is obtained, as shown in Figure 9.

4.3.2. Analysis of the Development Capability of Each Subsystem of the IoT Industry. The coefficients a11, a22, a33, and a44 are the self-development coefficients of the integration, technological innovation, structural adjustment, and organizational mechanism of the IoT industrial system. Among them, the self-development coefficient of the fusion subsystem a11 $>0$ indicates that the fusion subsystem has the ability of self-development. The main reason is that the basic data of the fusion subsystem is showing a gentle 
upward trend, which also verifies the upward trend of the orderliness of the fusion subsystem. The self-development coefficient of the technological innovation subsystem a22 $>0$ indicates that the subsystem has a good self-development ability, which verifies the rapid development trend of the technological innovation subsystem's orderliness, and the development level of the system's orderliness is higher than the other three subsystems. The order of science and technology expenditure and the order parameters of intangible assets has shown a rapid upward trend, mainly due to the continuous development of technological innovation in the IoT. The self-development coefficient of the structural adjustment mechanism subsystem a $33>0$ indicates that the structural adjustment mechanism subsystem has the ability to develop itself, but this ability is relatively weak. The organization mechanism subsystem a $44>0$ indicates that the organization mechanism subsystem currently has the ability to develop itself, and the order of system development is gradually improving. The gray model coefficients of each subsystem of the IoT industry are shown in Figure 10.

\section{Conclusion}

After establishing various mechanism models and solving them, this paper puts forward the conclusions of various mechanisms and application countermeasures based on the results of the solution and specially introduces a coordination manager who asks for the remaining system coordination gains to be responsible for the implementation of this series of coordination mechanisms. The system coordination manager should allocate the synergy gain to the members scientifically and induce the members to help each other through the incentive mechanism. At the same time, behavior supervision and information sharing supervision mechanisms are also needed. If the lack of supervision mechanism leads to poor service quality, customers will not choose the service provider of the network system but choose other IoTorganization model enterprise services, and the operational stability of the IoT organization model network system will also be affected. After the orderliness of the structural adjustment mechanism reached its lowest point, it began to rise gradually. In the structural adjustment mechanism, the labor structure has the largest impact, followed by the technical structure. Then increasing the introduction of talents and expanding intellectual support will play a role in improving the orderliness of the structural adjustment mechanism. The orderliness of the organizational mechanism shows a downward trend after reaching the peak, indicating that the organizational mechanism that can lead the development of the IoT industry is not yet perfect. Improving the performance of industrial organizations and improving profitability have a greater effect on the orderliness of organizational mechanisms. We analyze the development capabilities of the subsystems themselves and the relationship between them through the gray dynamic correlation analysis model. At present, each subsystem has the self-development capability, and the fusion subsystem and the technological innovation subsystem have a unidirectional weak synergy relationship.

\section{Data Availability}

Data sharing is not applicable to this paper as no datasets were generated or analyzed during the current study.

\section{Conflicts of Interest}

The authors declare that there are no conflicts of interest.

\section{References}

[1] J. Liu, H. Nishiyama, N. Kato, and J. Guo, "On the outage probability of device-to-device-communication-enabled multichannel cellular networks: an RSS-threshold-based perspective," IEEE Journal on Selected Areas in Communications, vol. 34, no. 1, pp. 163-175, 2016.

[2] V. K. Chawla, A. K. Chanda, S. Angra, and S. Rani, "Effect of nature-inspired algorithms and hybrid dispatching rules on the performance of automatic guided vehicles in the flexible manufacturing system," Journal of the Brazilian Society of Mechanical Sciences and Engineering, vol. 41, no. 10, p. 391, 2019.

[3] J. Myers, A. Savanth, R. Gaddh, D. Howard, P. Prabhat, and D. Flynn, "A subthreshold ARM cortex-M0+ subsystem in 65 nm CMOS for WSN applications with 14 power domains, $10 \mathrm{~T}$ SRAM, and integrated voltage regulator," IEEE Journal of Solid-State Circuits, vol. 51, no. 1, pp. 31-44, 2016.

[4] A. Lamperski and J. C. Doyle, "The \$ $\{\mid c a l ~ H\} \_\{2\}$ \$ control problem for quadratically invariant systems with delays," IEEE Transactions on Automatic Control, vol. 60, no. 7, pp. 1945-1950, 2015.

[5] U. Orozco-Rosas, K. Picos, and O. Montiel, "Hybrid path planning algorithm based on membrane pseudo-bacterial potential field for autonomous mobile robots," IEEE Access, vol. 7, pp. 156787-156803, 2019.

[6] Y. He, N. Zhao, and H. Yin, "Integrated networking, caching, and computing for connected vehicles: a deep reinforcement learning approach," IEEE Transactions on Vehicular Technology, vol. 67, no. 1, pp. 44-55, 2018.

[7] S. Pape and K. Rannenberg, "Applying privacy patterns to the Internet of things' (IoT) architecture," Mobile Networks and Applications, vol. 24, no. 3, pp. 925-933, 2019.

[8] H. Reyserhove and W. Dehaene, "A differential transmission gate design flow for minimum energy sub-10-pJ/cycle ARM cortex-M0 MCUs," IEEE Journal of Solid-State Circuits, vol. 52, no. 7, pp. 1904-1914, 2017.

[9] S. Sabau and N. C. Martins, "Youla-like parametrizations subject to QI subspace constraints," IEEE Transactions on Automatic Control, vol. 59, no. 6, pp. 1411-1422, 2014.

[10] Y. Lu, C. Cheng, J. Yang, and G. Gui, "Improved hybrid precoding scheme for mmWave large-scale MIMO systems," IEEE Access, vol. 7, pp. 12027-12034, 2019.

[11] Y. Zhao, X. Liu, G. Wang, S. Wu, and S. Han, "Dynamic resource reservation based collision and deadlock prevention for multi-AGVs," IEEE Access, vol. 8, pp. 82120-82130, 2020.

[12] N. Zhang, P. Yang, J. Ren, D. Chen, L. Yu, and X. Shen, "Synergy of big data and 5G wireless networks: opportunities, approaches, and challenges," IEEE Wireless Communications, vol. 25, no. 1, pp. 12-18, 2018.

[13] V. Casola, A. De Benedictis, M. Erascu, J. Modic, and M. Rak, "Automatically enforcing security SLAs in the cloud," IEEE Transactions on Services Computing, vol. 10, no. 5, pp. 741755, 2017. 
[14] S. Paul, V. Honkote, R. G. Kim et al., "A sub-cm3 energyharvesting stacked wireless sensor node featuring a nearthreshold voltage IA-32 Microcontroller in 14-nm tri-gate CMOS for always-ON always-sensing applications," IEEE Journal of Solid-State Circuits, vol. 52, no. 4, pp. 961-971, 2017.

[15] F. Lin, M. Fardad, and M. R. Jovanovic, "Design of optimal sparse feedback gains via the alternating direction method of multipliers," IEEE Transactions on Automatic Control, vol. 58, no. 9, pp. 2426-2431, 2013.

[16] A. Asef-Vaziri and M. Kazemi, "Covering and connectivity constraints in loop-based formulation of material flow network design in facility layout," European Journal of Operational Research, vol. 264, no. 3, pp. 1033-1044, 2018.

[17] J. Ni, X. Lin, and X. S. Shen, "Efficient and secure serviceoriented authentication supporting network slicing for 5Genabled IoT," IEEE Journal on Selected Areas in Communications, vol. 36, no. 3, pp. 644-657, 2018.

[18] F. Conti, R. Schilling, P. D. Schiavone et al., "An IoT endpoint system-on-chip for secure and energy-efficient near-sensor analytics," IEEE Transactions on Circuits and Systems I: Regular Papers, vol. 64, no. 9, pp. 2481-2494, 2017.

[19] K. Dvijotham, E. Todorov, and M. Fazel, "Convex structured controller design in finite horizon," IEEE Transactions on Control of Network Systems, vol. 2, no. 1, pp. 1-10, 2015.

[20] W. Xing, L. Peihuang, Y. Jun, Q. Xiaoming, and T. Dunbing, "Intersection recognition and guide-path selection for a vision-based AGV in a bidirectional flow network," International Journal of Advanced Robotic Systems, vol. 11, no. 3, p. 39, 2014.

[21] G. Gui, H. Huang, Y. Song, and H. Sari, "Deep learning for an effective nonorthogonal multiple access scheme," IEEE Transactions on Vehicular Technology, vol. 67, no. 9, pp. 8440-8450, 2018.

[22] R. Ranchal, B. Bhargava, P. Angin, and L. B. Othmane, "Epics: a framework for enforcing security policies in composite web services," IEEE Transactions on Services Computing, vol. 12, no. 3, pp. 415-428, 2019. 